\title{
A search for young stellar objects as possible counterparts of Fermi sources
}

\section{Pere Munar-Adrover}

Departament d'Astronomia i Meteorologia, Institut de Ciències del Cosmos (ICC), Universitat de Barcelona (IEEC-UB)

E-mail: pmunar@am.ub.es

\section{Josep M. Paredes}

Departament d'Astronomia i Meteorologia, Institut de Ciències del Cosmos (ICC), Universitat de Barcelona (IEEC-UB)

E-mail: jmparedes@ub.edu

\section{Gustavo E. Romero}

Instituto Argentino de Radioastronomía, Universidad de La Plata

E-mail: romerodiar.unlp.edu.ar

\begin{abstract}
Massive protostars have associated bipolar outflows which can produce strong shocks when interact with the surrounding medium. Some theoretical models predict that, under some conditions, particle acceleration at relativistic velocities can occur leading to gamma ray emission. In order to identify young stellar objects (YSO) that might emit gamma rays, we have crossed the Fermi First Year Catalog with catalogs of known YSOs, obtaining a set of candidates by spatial correlation. We have conducted Monte Carlo simulations to find the probability of chance coincidence. Our results indicate that $\sim 70 \%$ of the candidates should be gamma-ray sources with a confidence of $\sim 5 \sigma$.
\end{abstract}

25th Texas Symposium on Relativistic Astrophysics - TEXAS 2010

December 06-10, 2010

Heidelberg, Germany 


\section{Introduction}

Massive YSOs show collimated outflows, and thermal radiation has been detected up to distances of $10^{16}-10^{18} \mathrm{~cm}$ from the central star. These are strongly supersonic jets and in some cases, non-thermal radio lobes have been detected at distances of $Z_{j} \sim 1 \mathrm{pc}$ [1]. These radio lobes are probably generated by strong terminal shocks of the jets, which also ionize the shocked material. The possibility of YSOs to be $\gamma$-ray emitters has already been discussed in [2] and [3].

The action of the jet head on the external medium leads two shocks, one moving in the cloud material and one moving in the jet itself: the bow shock and the reverse shock, respectively. The observed non-thermal radio emission would be generated at the shocks where the particles are accelerated.

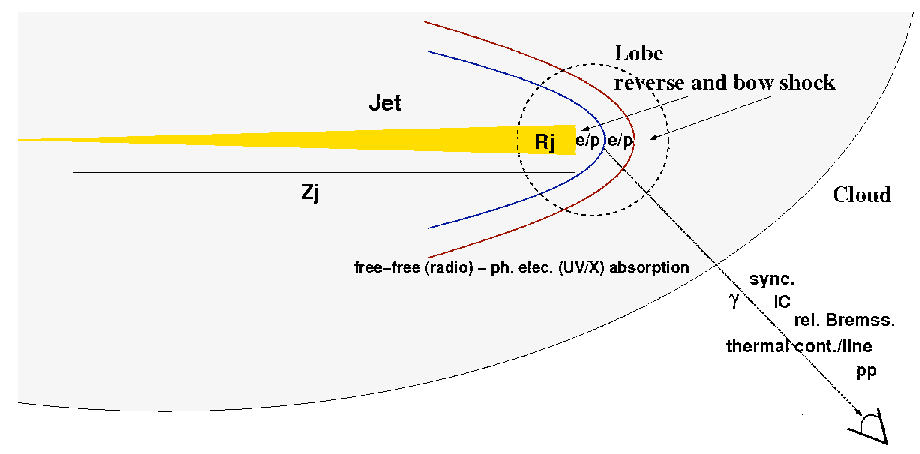

Figure 1: Diagram of the termination region of the jet of a massive YSO. The two shocks are represented. Particles can be accelerated in these shocks and produce non-thermal radiation via interaction with the surrounding matter, the radiation field and the magnetic field. [3]

\subsection{Catalogs}

In this work we have crossed two catalogs in order to get two-dimensional spatial coincidences: the Fermi Large Area Telescope First Catalog and the Red MSX Source (RMS) survey.

The Fermi Large Area Telescope First Catalog [⿰亻也 contains the detected sources during the first 11 months of the science phase of the mission, which began on 2008 August 4. This catalog contains 1451 gamma-ray sources detected and characterized in the $100 \mathrm{MeV}$ to $100 \mathrm{GeV}$ range with a typical position uncertainty of $\sim 6^{\prime}$. Excluding the firm identifications from the original sample we get 1392 sources. Most of them are located on the galactic plane (see Figure 2).

The Red MSX Source (RMS) survey is an ongoing multi-wavelength (from radio to infrared) observational program with the objective of providing a well-selected sample of MYSOs in the entire Galaxy [5]. About 2000 MYSO candidates have been identified by comparing the colours of MSX and 2MASS point sources (at 8, 12, 14 and $23 \mu \mathrm{m}$ ) with those of well known MYSOs. The survey also uses high resolution radio continuum observations at $6 \mathrm{~cm}$ obtained with the VLA in the northern hemisphere, and at $3.6 \mathrm{~cm}$ and $6 \mathrm{~cm}$ with ATCA in the southern hemisphere, that help to distinguish between genuine MYSOs and other types of objects, such as ultra compact HII regions, evolved stars or planetary nebulae, that contaminate the sample. In addition to these targeted observations, archival data of previous VLA survey of the inner Galaxy has been used. 
Figure 2: Distribution of First Fermi Catalog sources in galactic latitude.

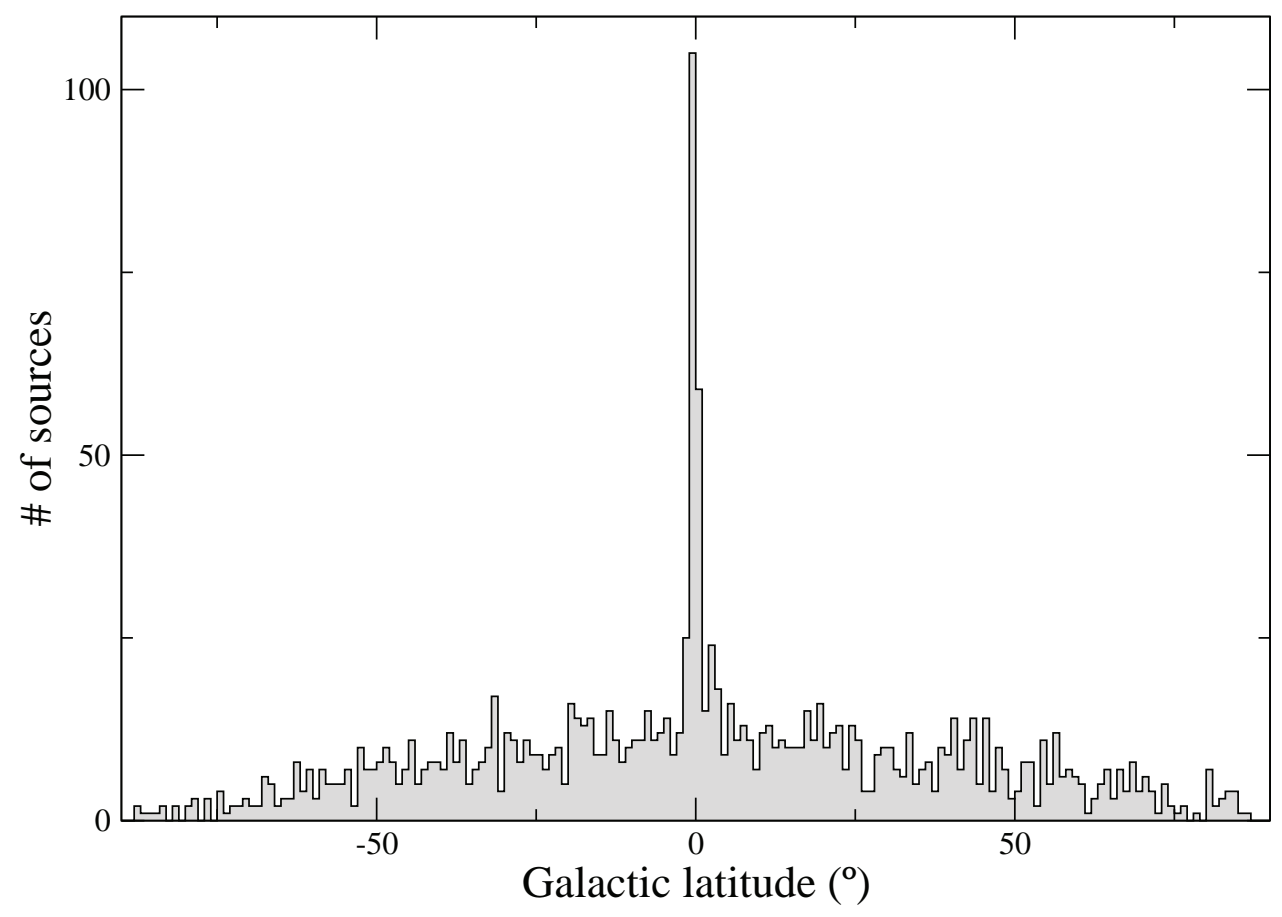

This ongoing program has provided a sample of 637 well-identified MYSOs until now, which have been used in our work.

\section{Monte Carlo analysis}

We have simulated a large number of sets of Fermi sources with the Monte Carlo method, in order to estimate the chance coincidence probability. Specifically, we have simulated 1500 populations of 1392 Fermi sources, through rotations on the celestial sphere, displacing a source with original galactic coordinates $(l, b)$ to a new position $\left(l_{0}, b_{0}\right)$ in bins of $1^{\circ}$ and $2^{\circ}$, using the same algorithm used in [6], keeping the distribution of the gamma-ray sources in the galactic latitude coordinate. The separation in the plane of the sky between the Fermi source and the YSO is calculated in each case using the statistical parameter $R$ [7], which takes into account the position uncertainties in equatorial coordinates of each compared source:

$$
R=\sqrt{\frac{(\Delta \alpha \cos \delta)^{2}}{\sigma_{\alpha_{i}}^{2}+\sigma_{\alpha_{j}}^{2}}+\frac{\Delta \delta^{2}}{\sigma_{\delta_{i}}^{2}+\sigma_{\delta_{j}}^{2}}}
$$


where $\sigma_{\alpha_{i}}, \sigma_{\delta_{i}}$ is the uncertainty in the equatorial coordinates of the source, and $i$ and $j$ are Fermi and RMS sources, respectively.

\section{Results}

Our cross identification study shows that 12 Fermi sources are positionally coincident with 22 YSOs (see Table 2). In this table we present, from left to right, the Fermi source name, its J2000 equatorial coordinates, its positional uncertainty, the spectral $\gamma$-ray index, the energy flux (E $>100$ $\mathrm{MeV}$ ), the YSO name, its J2000 equatorial coordinates, the angular distance between the two compared sources, the distance to the YSO, its IR luminosity and the mass of the star forming region where it is embedded. The results from the Monte Carlo study are shown in Table 1. For the case of displacing the gamma-ray sources in $1^{\circ}$-bins, the Monte Carlo simulations return an average of coincidences of $4.4 \pm 2.2$ sources. This is the number of chance coincidences. This result means that from 12 real coincidences, 7.6 ( $\sim 63 \%$ of the total coincidences with a confidence level of $\sim 4 \sigma$ ) might be associated with a probability of chance coincidence of $1.8 \times 10^{-4}$. In a similar way, in the case of moving the Fermi sources in $2^{\circ}$-bins, we obtain a chance coincidence number of 3.6 \pm 1.8 , which means that 8.4 of the 12 coincidences should be associated $(\sim 70 \%$ at $\sim 5 \sigma$ confidence level) with a probability of $5.6 \times 10^{-6}$ of being chance coincidences.

Table 1: Statistical results obtained from simulations with a random distribution in galactic longitude.

\begin{tabular}{ccccc}
\hline \hline $\begin{array}{c}\text { Actual } \\
\text { coincidence }\end{array}$ & $\begin{array}{c}\text { Simulated } \\
1^{\circ} \text {-bin }\end{array}$ & $\begin{array}{c}\text { Probability } \\
1^{\circ}\end{array}$ & $\begin{array}{c}\text { Simulated } \\
2^{\circ} \text {-bin }\end{array}$ & $\begin{array}{c}\text { Probability } \\
2^{\circ}\end{array}$ \\
\hline 12 & $4.4 \pm 2.0$ & $1.8 \times 10^{-4}$ & $3.6 \pm 1.8$ & $5.6 \times 10^{-6}$ \\
\hline
\end{tabular}

\section{Acknowledgments}

The authors acknowledge support of the Spanish Ministerio de Ciencia e Innovación (MICINN) under grant AYA2010-21782-C03-01. G.E.R. also acknowledges support of PIP 0078 (CONICET).

\section{References}

[1] Garay G., Brooks K.J., Mardones D., and Norris R.P., 2003, ApJ, 587, 739-747

[2] Araudo A.T., Romero G.E., Bosch-Ramon V., and Paredes J.M., 2007, A\&A, 476, 1289-1295

[3] Bosch-Ramon V., Romero G.E., Araudo A.T. and Paredes J.M., 2010, A\&A, 511, A8

[4] Abdo A.A. et al., 2010, ApJ Supplement Series, 188, 405-436

[5] Urquhart J.S., Horae M.G., Purcell C.R. et al. , 2009, A\&A, 501, 539-551

[6] Romero G.E., Benaglia P. and Torres D.F., 1999, A\&A, 348, 868-876

[7] Allington-Smith J.R., Perryman M.A.C., Longair M.S. et al., 1982, MNRAS, 201, 331-344 
Table 2: Positional coincidence of Fermi sources with MYSOs.

\begin{tabular}{|c|c|c|c|c|c|c|c|c|c|c|c|c|}
\hline $\begin{array}{l}\text { Fermi Name } \\
(1 \mathrm{FGL})\end{array}$ & $\begin{array}{l}\text { RA } \\
\left({ }^{\circ}\right)\end{array}$ & $\begin{array}{l}\begin{array}{l}\text { Dec } \\
\left({ }^{\circ}\right)\end{array} \\
\end{array}$ & $\begin{array}{l}95 \% \text { Semi } \\
\text { Major Axis }\left({ }^{\circ}\right)\end{array}$ & $\begin{array}{c}\text { Spectral Index } \Gamma \\
\left(F \propto E^{-\Gamma}\right)\end{array}$ & $\begin{array}{c}\text { Flux }(\mathrm{E}>100 \mathrm{MeV}) \\
\times 10^{-11} \mathrm{erg} \mathrm{cm}^{-2} \mathrm{~s}^{-1}\end{array}$ & MSX Name & $\begin{array}{l}\text { RA } \\
\left({ }^{\circ}\right)\end{array}$ & $\begin{array}{l}\text { Dec } \\
\left({ }^{\circ}\right)\end{array}$ & $\begin{array}{l}\Delta \theta \\
\left.{ }^{\circ}\right)\end{array}$ & 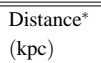 & $\begin{array}{l}L_{\text {bool }}^{*} \\
\left(\times 10^{3} \mathrm{~L}_{\odot}\right)\end{array}$ & $\begin{array}{l}\text { Mass } \\
\left(M_{\odot}\right)\end{array}$ \\
\hline \multirow[t]{3}{*}{$\mathrm{J} 0541.1+3542$} & 85.2805 & 35.7091 & 0.1397 & $2.41 \pm 0.13$ & $1.6 \pm 0.5$ & G173.6328+02.8064 & 85.27929 & +35.82633 & 0.12 & $1.6^{a}$ & $4.8^{d}$ & \\
\hline & & & & & & G173.6339+02.8218 & 85.29592 & +35.83380 & 0.13 & $1.6^{a}$ & $3.2^{e}$ & \\
\hline & & & & & & G173.6882+02.7222 & 85.22758 & +35.73558 & 0.05 & $1.6^{a}$ & - & \\
\hline J0647.3+0031 & 101.8417 & 0.5289 & 0.2150 & $2.41 \pm 0.11$ & $1.9 \pm 0.5$ & G212.0641-00.7395 & 101.80567 & +0.43514 & 0.10 & & $25^{f}$ & \\
\hline J1256.9-6337 & 194.2474 & -63.6212 & 0.1955 & $2.26 \pm 0.12$ & $4.9 \pm 1.1$ & G303.5990-00.6524 & 194.35546 & -63.51650 & 0.12 & $11.3^{b}$ & $8.3^{f}$ & \\
\hline J1315.0-6235 & 198.7635 & -62.5971 & 0.1860 & $2.31 \pm 0.12$ & $6.9 \pm 0.0$ & G305.4840+00.2248 & 198.40016 & -62.53708 & 0.18 & $3.6^{b} 乙$ & $3.8^{f}$ & \\
\hline$J 1651.5-4602$ & 252.8831 & -46.0340 & 0.2258 & $2.21 \pm 0.07$ & $13.9 \pm 3.4$ & G339.8838-01.2588 ${ }^{1}$ & 253.01942 & -46.14267 & 0.14 & $2.6^{b}$ & $21.0^{f}$ & \\
\hline \multirow[t]{2}{*}{ J1702.4-4147 } & 255.6039 & -41.7859 & 0.0800 & $2.39 \pm 0.07$ & $8.7 \pm 2.0$ & G344.4257+00.0451B & 255.53674 & -41.78303 & 0.05 & $5.0^{b}$ & $15.0^{f}$ & \\
\hline & & & & & & G344.4257+00.0451C & 255.53587 & -41.78617 & 0.05 & $5.0^{b}$ & $15.0^{f}$ & \\
\hline$J 1846.8-0233$ & 281.7001 & -2.5628 & 0.1262 & $2.21 \pm 0.06$ & $9.3 \pm 2.3$ & G030.1981-00.1691 & 281.76274 & -2.51003 & 0.08 & $7.4^{b}$ & $29.0^{f}$ & \\
\hline \multirow[t]{2}{*}{ J1848.1-0145 } & 282.0470 & -1.7605 & 0.0859 & $2.23 \pm 0.04$ & $9.5 \pm 3.2$ & G030.9726-00.1410 & 282.09178 & -1.80842 & 0.07 & $5.7^{b}$ & $3.9^{f}$ & $1.9 \times 10^{3} \mathrm{~g}$ \\
\hline & & & & & & G030.9959-00.0771 & 282.04516 & $-1,75808$ & 0.0044 & $5.7^{b}$ & $5.1^{f}$ & $1.9 \times 10^{3} \mathrm{~g}$ \\
\hline \multirow[t]{6}{*}{$\mathrm{J} 1853.1+0032$} & 283.2884 & 0.5366 & 0.5207 & $2.18 \pm 0.07$ & $5.7 \pm 1.7$ & G032.8205-00.3300 & 282.04436 & -1.75703 & 0.34 & $5.1^{b}$ & $17.0^{f}$ & \\
\hline & & & & & & G033.3891+00.1989 & 282.89092 & +0.49750 & 0.40 & & $11.0^{f}$ & \\
\hline & & & & & & G033.3933+00.0100 & 283.06109 & +0.41528 & 0.26 & $6.8^{b}$ & $7.9^{e}$ & \\
\hline & & & & & & G033.5237+00.0198 & 283.11179 & +0.53569 & 0.34 & & $7.9^{e}$ & \\
\hline & & & & & & G034.0126-00.2832 & 283.60437 & +0.83239 & 0.43 & $13.3^{b}-$ & $34.0^{f}$ & \\
\hline & & & & & & G034.0500-00.2977 & 283.63454 & +0.85914 & 0.47 & $13.3^{b} \subset$ & $24.0^{f}$ & \\
\hline \multirow[t]{2}{*}{$\mathrm{J} 1925.0+1720$} & 291.2748 & 17.3485 & 0.1443 & $2.28 \pm 0.12$ & $2.4 \pm 1.0$ & G052.2025+00.7217A & 291.24933 & +17.42169 & 0.08 & $10.2^{b} \smile$ & $15.0^{f}$ & \\
\hline & & & & & & G052.2078+00.6890 & 291.28553 & +17.41317 & 0.07 & $10.2^{b}$ & & \\
\hline $\mathrm{J} 1943.4+2340$ & 295.8667 & 23.6815 & 0.1118 & $2.23 \pm 0.11$ & $2.6 \pm 0.7$ & G059.7831+00.0648 & 295.79680 & +23.73433 & 0.08 & $2.2^{a} \cup$ & $6.8^{f}$ & 840 and $190^{h}$ \\
\hline J2040.0+4157 & 310.0154 & 41.9533 & 0.1970 & $2.66 \pm 0.06$ & $7.9 \pm 1.2$ & $\mathrm{G} 081.5168+00.1926$ & 309.99066 & +41.98739 & 0.04 & ${ }_{1.7^{c}} \bigcirc$ & $0.704^{f}$ & \\
\hline
\end{tabular}

